\title{
Anaesthesia for radiotherapy in paediatric oncology-a retrospective observational study in an Asian population
}

\author{
Lydia W. Li ${ }^{1,2,3 \#}$, Gail WY Chua ${ }^{4 \#}$, Koh Wenjun $^{1}$, Choon Looi Bong ${ }^{5}$ \\ ${ }^{1}$ Yong Loo Lin School of Medicine, National University of Singapore, Singapore; ${ }^{2}$ Department of Anaesthesia \& Intensive Care, Changi General \\ Hospital, Singapore; ${ }^{3}$ Duke-NUS Medical School, Singapore; ${ }^{4}$ Division of Radiation Oncology, National Cancer Centre, Singapore; ${ }^{5}$ Department of \\ Paediatric Anaesthesia, KK Women's and Children's Hospital, Singapore \\ Contributions: (I) Conception and design: LW Li, GWY Chua, CL Bong; (II) Administrative support: All authors; (III) Provision of study materials \\ or patients: LW Li, GWY Chua, CL Bong; (IV) Collection and assembly of data: All authors; (V) Data analysis and interpretation: All authors; (VI) \\ Manuscript writing: All authors; (VII) Final approval of manuscript: All authors. \\ "These authors contributed equally to this work. \\ Correspondence to: Dr. Lydia W. Li. Department of Anaesthesia \& Surgical Intensive Care, Changi General Hospital, 2 Simei Street 3, Singapore \\ 529889, Singapore. Email: lydia.li.w@singhealth.com.sg.
}

Background: Immobilisation in radiotherapy treatment is especially important as many paediatric tumours are located near critical organs. Although the external beam radiotherapy treatment process itself is painless, the immobilization devices used may cause anxiety and discomfort in children who are too young to understand and co-operate. Hence, anaesthesia or sedation is unavoidable in such cases. This descriptive study aims to evaluate the demographics, methods and outcomes of paediatric radiotherapy patients in our Asian context.

Methods: This is a single-institution, retrospective observational study in a tertiary hospital with more than a decade of experience in paediatric radiotherapy. Data collected include age, gender, race, ASA status, oncology diagnosis, site of radiotherapy, position of patient during procedure, need for sedation or anaesthesia, anaesthetic drugs used, airway devices, use of premedications, use of antiemetics, and incidence and type of complications. We also analysed the association between various factors (e.g., age, radiotherapy treatment site) and usage of anaesthesia. Statistical analysis was carried out using a multivariable model.

Results: Between January 2006 and December 2017, 434 paediatric patients underwent radiotherapy, with a total of 10,357 discrete radiotherapy sessions. In 1,276 radiotherapy sessions (11.8\%) either general anaesthesia or sedation was required. The need for anaesthesia was highly age-dependent - all patients under 3 years of age required anaesthesia, whereas anaesthesia was rarely needed in patients above 7 years of age. Our institution had a higher prevalence of inhalational agent usage as opposed to intravenous agent usage. Complication rate was relatively low.

Conclusions: This is the first retrospective review performed in our country on paediatric patients receiving anaesthesia during radiotherapy, evaluating patients' demographic data, and type of anaesthesia and radiotherapy techniques used. We found that patients' requirement for anaesthesia decreases in an age-dependent fashion, in line with existing literature. Our reported complication rate during anaesthesia sessions was low, testament to the high standards and safety of our techniques.

Keywords: Paediatrics; anaesthesia; radiation oncology; radiotherapy

Submitted Jan 02, 2020. Accepted for publication Dec 31, 2020.

doi: $10.21037 / \mathrm{cco}-20-3$

View this article at: http://dx.doi.org/10.21037/cco-20-3 


\section{Introduction}

Radiotherapy treatment in paediatric cancer patients, for curative or palliative purposes, poses significant clinical and technical challenges. These challenges involve both safe delivery of radiotherapy treatment and delivery of anaesthesia. For safe delivery of radiotherapy, patients must be immobilized during both simulation and treatment, and the set-up must be reproducible throughout the treatment period to avoid unnecessary radiation exposure. The need for immobilization devices often causes anxiety and fear in children who are too young to understand and cooperate, hence necessitating anaesthesia or sedation.

However, the delivery of anaesthesia in children undergoing radiotherapy is often not a straightforward process. Firstly, paediatric patients of different age groups have unique anatomical and physiological variations that require the expertise of a paediatric anaesthetist. Furthermore, radiotherapy simulation and treatment are usually done in remote settings outside of the operating theatre where the anaesthetist's resources could be limited. Certain aspects of radiotherapy simulation and immobilization devices may pose an additional challenge to the anaesthetist-for instance, some patients need to be in the prone instead of supine position, and patients receiving radiotherapy to the head and neck require a shell that covers the face. Hence, airway management in these situations can be a challenging process.

Despite the high frequency of paediatric oncology radiotherapy treatment sessions at our centre, there exists a knowledge gap with regards to the methods and outcomes of our anaesthetic practice for paediatric radiotherapy patients in the past eleven years. Our primary objective was to characterise the demographics of our patients. We also aimed to determine the incidence of anaesthesiarelated complications at our institution, and to report the anaesthetic and radiotherapy techniques used.

We present the following article in accordance with the STROBE reporting checklist (available at http://dx.doi. org/10.21037/cco-20-3).

\section{Methods}

This is a retrospective observational study conducted in a single tertiary institution in Singapore. The study was conducted in accordance with the Declaration of Helsinki (as revised in 2013). The study was reviewed and approved by the Singhealth Centralized Institutional Review Board
(CIRB Ref 2018/2281) and individual consent for this retrospective study was waived.

We retrospectively analysed the medical records of all paediatric oncology patients from age 0 to 18 years old in a single tertiary institution who underwent radiotherapy between January 2006 and December 2017. Our inclusion criteria were all patients 18 years old and under who underwent any form of radiotherapy treatment for malignancy between January 2006 and December 2017 in our institution. Patients who did not complete the whole course of radiotherapy, turned 19 years old during radiotherapy, or received radiotherapy for non-malignant conditions (e.g., keloid) and those with missing data were excluded from our study. Data collected included age, gender, race, ASA status, oncology diagnosis, site of radiotherapy, position of patient during radiotherapy, need for sedation or anaesthesia, anaesthetic drugs used, airway devices, use of premedications, use of antiemetics and incidence and type of complications.

\section{Statistical analysis}

We presented patient characteristics in percentages (Table 1). We also analysed the association between various factors (e.g., age, radiotherapy treatment site) and usage of anaesthesia. Statistical analysis was done using a multivariable logistic regression model. Variable selection was performed using the backward elimination method, by optimizing AIC. Odds ratios (OR) and the corresponding $95 \%$ confidence intervals are presented, with odds ratios of $>1$ representing increased association with anaesthesia usage. $P$ values of $<0.05$ were deemed statistically significant.

\section{Results}

Between January 2006 and December 2017, 434 paediatrics patients underwent radiotherapy, with a total of 10,357 discrete radiotherapy sessions. Of these, 1,220 sessions $(11.8 \%)$ required general anaesthesia and 56 required sedation $(0.054 \%)$. The median age of the patients at radiotherapy session was 10 years (IQR, 6-15 years), while for those who required anaesthesia, the median age was 3.9 years.

In terms of radiotherapy technique, $87.5 \%$ of the patients were simulated using CT and $12.5 \%$ using conventional X-ray simulation. $92.0 \%$ of the patients were simulated and treated supine, $8.0 \%$ in the prone position. The most common site of treatment was cranium and craniospinal 
irradiation, followed by abdomen, extremities, spine, head and neck, thorax, pelvis, and total body irradiation/total nodal irradiation. Total treatment doses ranged from 2-66 Gy.

In a subgroup analysis of radiotherapy sessions which required support by Anaesthesia, the median age was 3.9 years with a standard deviation of 2.3 years. The minimum age was 0.8 years while the maximum was 15.8 years.

Sixty-one-point-five percent of patients were males, while $38.5 \%$ were female. Fifty-three-point-three percent were Chinese, 20.1\% Malay and 8.3\% Indian/Others. In terms of ASA status, $2.3 \%$ of patients were classified as ASA 1, 48.5\% ASA 2, 46.3\% ASA 3 and 2.9\% ASA 4.

Of the patients that required Anaesthetist support, 95.6\% of cases were eventually done under general anaesthesia, while $4.4 \%$ of cases were done under sedation. Inhalational induction with sevoflurane was used in $81.3 \%$ of these cases, while intravenous propofol was used in $16.2 \%$ of cases. $90.9 \%$ of GA cases were maintained with sevoflurane, while $1.4 \%$ of cases used total intravenous anesthesia. In terms of airway device, $74.4 \%$ used a laryngeal mask airway, $1.8 \%$ secured the airway using an endotracheal tube, while $0.2 \%$ held a face mask.

We also looked at the rates of premedication-this was used in 21.8\% of cases with Anaesthetist support. In 24.1\% of cases, prophylactic antiemetics were administered.

Out of a total 1,276 radiotherapy sessions that required support by an Anaesthetist, the incidence of reported complications was $4(0.3 \%)$-all of which were respiratory in nature, 3 were laryngospasm and 1 apnea.

Separately, we analysed the factors associated with anaesthesia usage using a multivariable logistic regression model. Variable selection was performed using the backward elimination method, by optimizing AIC. We found that increasing age was significantly associated with decreased usage of anaesthesia, OR 0.28 (95\% CI: $0.21-$ $0.37), \mathrm{P}<0.001$. In addition, we found that radiotherapy to the following sites were significantly associated with usage of anaesthesia: cranium alone, OR 3.69 (95\% CI: 1.1912.06), $\mathrm{P}=0.026$, craniospinal irradiation, OR 34.18 (95\% CI: 6.19-217.97), $\mathrm{P}<0.001$, spine alone, OR 6.26 (95\% CI: $1.31-32.92), \mathrm{P}=0.024$.

A summary of our results is presented in Tables 1,2, and Figures 1,2.

\section{Discussion}

Immobilisation in radiotherapy treatment is especially important as many paediatric tumours are located near critical organs; for example intracranial tumours such as medulloblastoma and craniopharyngioma, or retinoblastoma. Although the external beam radiotherapy treatment process itself is painless, the immobilization devices used may cause anxiety and discomfort in children who are too young to understand and co-operate. Hence, anaesthesia or sedation is unavoidable in such cases.

Figure 1 shows the percentage of patients requiring anaesthesia support by age cohort. This is comparable to other studies in current available literature. McMullen et al. (1) found that $100 \%$ of patients aged 3 years old treated at a single centre between 2004-2013 had required general anaesthesia during radiotherapy, with requirements for anaesthesia after age 3 years old decreasing in an age dependent fashion. Approximately $10 \%$ of children aged 12 years or older still required anaesthesia or sedation.

As can be seen in our study, $100 \%$ of patients below the age of 3 years required anaesthesia support. The majority of our patients requiring anaesthesia support are less than or equal to 6 years of age. The older the patient, the less likely he/she required anaesthesia support for radiotherapy. $0 \%$ of our patients 16 years and older required anaesthesia support. The largest variability in the need for anaesthesia support was within an age cohort between the ages of 4 to 11 years old. We postulate that the behavioural programs targeted at this age group would have the highest yield in reducing the need for unnecessary anaesthesia. Such programs aim to educate, familiarise and reassure these children through the use of proper orientation to the environment, play therapy, positive reinforcement and distraction therapy. Previous studies have shown promising associations between the implementation of behavioural modification techniques and decreased need for anaesthesia for radiotherapy $(2,3)$. Indeed, a comprehensive familiarisation and play therapy programme, with caregivers and medical team on-board, may help in mitigating anxiety in all involved and reducing 'elective'/'on-demand' requests for anaesthesia from parents or doctors themselves $(4,5)$.

The challenges of providing Anaesthesia for paediatric radiotherapy are manifold. Paediatric patients have physiological and anatomical variations that require the expertise of a Paediatric Anaesthetist. Radiotherapy sessions are carried out in a remote setting- an environment that may be unfamiliar to the Anaesthetist and where trained assistance and equipment may be inaccessible. Airway devices may be restricted by the use of face shells used in head and neck cancers. To avoid radiation exposure, the patient must be left alone during the session and monitored 
Table 1 Patient characteristics

\begin{tabular}{|c|c|}
\hline Characteristics & Data \\
\hline Patients receiving radiotherapy & 434 \\
\hline Total radiotherapy sessions & 10,357 \\
\hline Sessions requiring general anaesthesia & $1,220(11.8 \%)$ \\
\hline Sessions requiring sedation & $56(0.054 \%)$ \\
\hline Median age at radiotherapy (years) & $10(\mathrm{IQR}, 6-15)$ \\
\hline \multicolumn{2}{|l|}{ Anaesthesia subgroup } \\
\hline Median age of patients requiring anaesthesia & 3.9 \\
\hline \multicolumn{2}{|l|}{ Gender (\%) } \\
\hline Male & 61.5 \\
\hline Female & 38.5 \\
\hline \multicolumn{2}{|l|}{ Race (\%) } \\
\hline Chinese & 53.3 \\
\hline Malay & 20.1 \\
\hline Indian/others & 8.3 \\
\hline \multicolumn{2}{|l|}{ ASA status (\%) } \\
\hline 1 & 2.3 \\
\hline 2 & 48.5 \\
\hline 3 & 46.3 \\
\hline 4 & 2.9 \\
\hline \multicolumn{2}{|l|}{ Anaesthesia technique-induction (\%) } \\
\hline Inhalational & 81.3 \\
\hline Intravenous & 16.2 \\
\hline \multicolumn{2}{|l|}{ Anaesthesia technique-maintenance (\%) } \\
\hline Inhalational & 90 \\
\hline Intravenous & 1.4 \\
\hline \multicolumn{2}{|l|}{ Airway device (\%) } \\
\hline LMA & 74.4 \\
\hline Endotracheal tube & 1.8 \\
\hline Face mask & 0.2 \\
\hline Use of premedications (\%) & 21.8 \\
\hline Use of antiemetics (\%) & 24.1 \\
\hline Reported complication rate (\%) & 0.3 \\
\hline \multicolumn{2}{|l|}{ Radiotherapy simulation technique (\%) } \\
\hline Conventional $\mathrm{X}$ ray SIM & 12.5 \\
\hline CT simulation & 87.5 \\
\hline
\end{tabular}

Table 1 (continued)
Table 1 (continued)

\begin{tabular}{lc}
\hline Characteristics & Data \\
\hline Position (\%) & 92 \\
Supine & 8 \\
Prone & \\
Treatment site (\%) & 30.7 \\
Cranium & 15.9 \\
Craniospinal & 20.5 \\
Abdomen & 6.8 \\
Extremities & 6.8 \\
Spine & 5.7 \\
Thorax & 4.5 \\
Head and Neck & 4.5 \\
Pelvis & 2.3 \\
Total body irradiation & 2.3 \\
Total nodal irradiation & \\
\hline
\end{tabular}

via a camera system, thus making monitoring of anaesthesia and depth of sedation even more challenging. As can be seen from our study, these group of patients have high ASA status as a result of their Oncological condition and attendant complications as well as complications from their treatments. Hence it is important to be cognizant of the challenges in administering Anaesthesia for this high-risk population. Yet, the literature surrounding the provision of Anaesthesia for Paediatric Radiotherapy remains scarce. There are no randomised controlled trials looking at anaesthetic techniques for Anaesthesia for Paediatric Radiotherapy and most studies are retrospective in nature. Our study is the first local study to evaluate our Anaesthetic practices in Paediatric Radiotherapy. Interestingly, unlike in available literature, the majority of our cases were induced and maintained with the inhalational agent Sevoflurane as opposed to an intravenous agent like propofol. A recent paper by Yildirım et al. (6) advocates the use of a propofolbased anaesthetic to reduce the rates of respiratory and cardiovascular complications in repeated anaesthesia for radiotherapy. Owusu-Agyemang et al. (7). also reported that propofol infusion can be used safely without the need for an airway device for patients undergoing prolonged proton therapy. We postulate that the reason for our differing practice could be that our patients are often outpatient and 
Table 2 Multivariable model—factors associated with anaesthesia usage

\begin{tabular}{lcc}
\hline & \multicolumn{1}{c}{ Multivariable model-anaesthesia usage } & P value \\
\cline { 2 - 3 } Age, mean (SD) & OR $(95 \%$ Cl) & $<0.001$ \\
Region & $0.28(0.21-0.37)$ & 0.026 \\
Abdo & 1 & $<0.001$ \\
Cranium & $3.69(1.19-12.06)$ & 0.679 \\
CSI & $34.18(6.19-217.97)$ & 0.392 \\
Extremities & $1.39(0.29-6.65)$ & 0.938 \\
H\&N & $2.47(0.35-20.74)$ & 0.442 \\
Others & $0.93(0.13-6.16)$ & 0.024 \\
Pelvis & $2.41(0.26-23.65)$ & 0.984 \\
Spine & $6.26(1.31-32.92)$ & 0.543 \\
Total body/Total nodal irradiation & $0.99(0.23-4.25)$ & $1.67(0.32-9.11)$ \\
Thorax/Breast & &
\end{tabular}

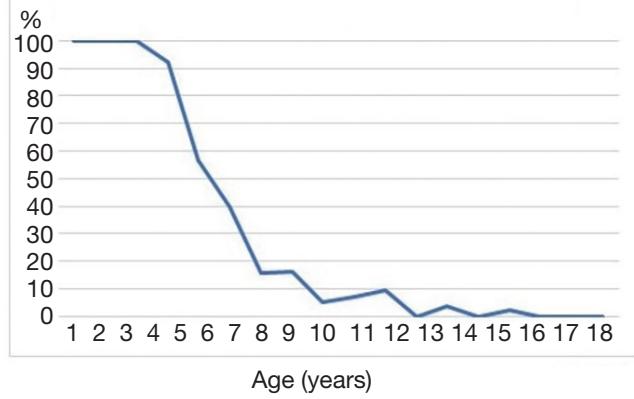

Figure 1 Percentage of children requiring GA/sedation during radiotherapy sessions.

do not have an intravenous access. Having an intravenous access for the almost-daily radiotherapy sessions is ideal but practical considerations of proper care of intravenous cannula by caregivers and the child's tolerance to the cannula deter frequent siting and re-siting of intravenous cannulas. The practice of using inhalational agents without an intravenous access is controversial in terms of safety. Our low complication rate is reassuring. However, this study has highlighted differences in our local practice compared to international practices and this warrants further consideration.

Our complication rate in this 11 -year audit is $0.3 \%$. This is lower than that reported in the current literature-in comparison, Anghelsescu et al. (8) reported a complication

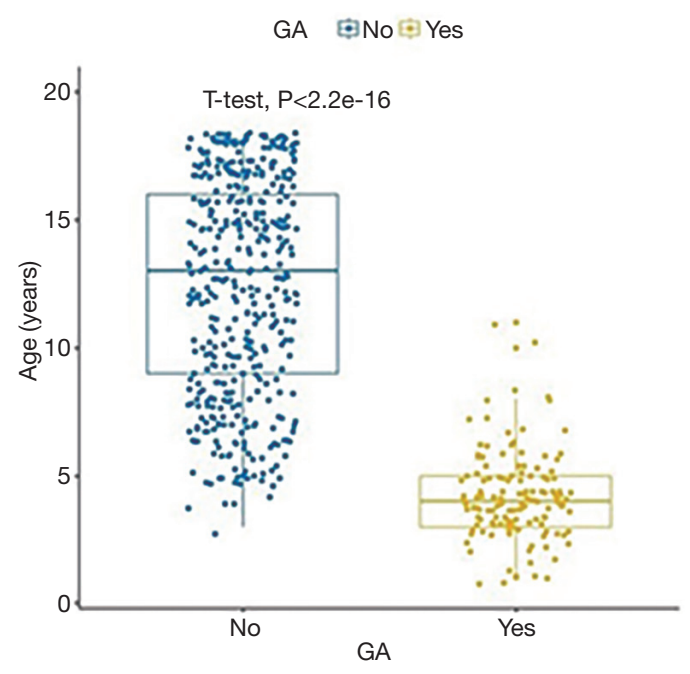

Figure 2 Box plot showing the relationship between age and usage of general anaesthesia.

rate of $1.3 \%$. Possible reasons for our low complication rate could be that patients were carefully selected prior to treatment to ensure fitness to receive anaesthesia. Furthermore, patients were closely monitored during set up, immobilisation and simulation, to ensure that the anaesthetic procedure would be well-tolerated during treatment. Finally, the low complication rate could be due in part to an element of under-reporting by the 
proceduralists themselves. All the complications reported were respiratory in nature. This is in keeping with the findings in current literature; for instance, Verma et al. (9) reported complications that were all respiratory based.

Several studies in the current literature (10) have looked at the demographic data and characteristics of patients undergoing radiotherapy under anaesthesia. However, to our knowledge, no such study has been done in our local context. We hope that our study would add to this body of literature and add insight into the association between our patients' ages and their need for anaesthetic support. This would allow a more focused use of our resources.

Going forward, we aim to minimise the use of anaesthesia in our patients as far as feasible. Our team of play therapists regularly work closely with preschool aged children to familiarise them with the radiotherapy treatment room, environment and immobilisation devices. This helps to minimise fear and help with co-operation between children and staff, contributing to a safe and efficient radiotherapy treatment process.

\section{Limitations}

Our study was a retrospective review of medical records.

\section{Conclusions}

This is the first retrospective review performed in our country on paediatric patients receiving anaesthesia during radiotherapy, evaluating patients' demographic data, and type of anaesthesia and radiotherapy techniques used. We found that patients' requirement for anaesthesia decreases in an age-dependent fashion, in line with existing literature. Our reported complication rate during anaesthesia sessions was low, testament to the high standards and safety of our techniques.

\section{Acknowledgments}

The authors would like to thank Ms. Zhou Siqin for her assistance with the statistical analysis.

Funding: None.

\section{Footnote}

Reporting Checklist: The authors have completed the STROBE reporting checklist. Available at http://dx.doi. org/10.21037/cco-20-3
Data Sharing Statement: Available at http://dx.doi. org/10.21037/cco-20-3

Conflicts of Interest: All authors have completed the ICMJE uniform disclosure form (available at http://dx.doi. org/10.21037/cco-20-3). The authors have no conflicts of interest to declare.

Ethical Statement: The authors are accountable for all aspects of the work in ensuring that questions related to the accuracy or integrity of any part of the work are appropriately investigated and resolved. The study was conducted in accordance with the Declaration of Helsinki (as revised in 2013). The study was reviewed and approved by the Singhealth Centralized Institutional Review Board (CIRB Ref 2018/2281) and individual consent for this retrospective study was waived.

Open Access Statement: This is an Open Access article distributed in accordance with the Creative Commons Attribution-NonCommercial-NoDerivs 4.0 International License (CC BY-NC-ND 4.0), which permits the noncommercial replication and distribution of the article with the strict proviso that no changes or edits are made and the original work is properly cited (including links to both the formal publication through the relevant DOI and the license). See: https://creativecommons.org/licenses/by-nc-nd/4.0/.

\section{References}

1. McMullen K, Hanson T, Bratton J, et al. Parameters of anesthesia/sedation in children receiving radiotherapy. Radiat Oncol 2015;10:65.

2. Halperin E. Radiation therapy for kids. (Videotape) Durham: Duke University Medical Center, Division of Educational Media Services; 1997.

3. Slifer KJ. A video system to help children cooperate with motion control for radiation treatment without sedation. J Pediatr Oncol Nurs 1996;13:91-7.

4. Tan L, Meakin G. Anaesthesia for the uncooperative child. Br J Anaesth 2010;10:48-52.

5. Dave N. Premedication and Induction of Anaesthesia in paediatric patients. Indian J Anaesth 2019;63:713-20.

6. Yıldırım İ, Çelik A, Bay S, et al. Propofol-based balanced anesthesia is safer in pediatric radiotherapy. J Oncol Pharm Pract 2019;25:1891-6.

7. Owusu-Agyemang P, Grosshans D, Arunkumar R, et al. Non-invasive anesthesia for children undergoing proton 
radiation therapy. Radiother Oncol 2014;111:30-4.

8. Anghelescu D, Burgoyne L, Liu W, et al. Safe Anesthesia for Radiation Therapy in Pediatric Oncology: the St. Jude Children's Research Hospital Experience, 2004-2006. Int J Radiat Oncol Biol Phys 2008;71:491-7.

9. Verma V, Beethe A, LeRiger M, et al. Anesthesia

Cite this article as: Li LW, Chua GW, Wenjun K, Bong CL. Anaesthesia for radiotherapy in paediatric oncology-a retrospective observational study in an Asian population. Chin Clin Oncol 2021;10(3):24. doi: 10.21037/cco-20-3 complications of pediatric radiation therapy. Pract Radiat Oncol 2016;6:143-54.

10. Fortney J, Halperin E, Hertz C, et al. Anesthesia for pediatric external beam radiation therapy. Int J Radiat Oncol Biol Phys 1999;44:587-91. 